\title{
Sachwortverzeichnis Band 6,1996
}

Verhaltenstherapie

Aggression 114 Agoraphobie 114 Angst 288

Atopische Dermatitits, psychische Faktoren 276

Biofeedback 143 Biographische Analyse 4244

Chronischer Schmerz 143

Depression 288

Diagnostik und Intervention 3170 Differentialdiagnosen 2100 Doppeldiagnose 4201

Ecstasy 4222

Effektgrößen 14

Einsatz von Kategoriensystemen 3135

Erythrophobie 133

Evaluation 4201

Forschung und Praxisbeziehung 3170

Gilles-de-la-Tourette-Syndrom 2100 Gruppentherapie 3143

Halluzinogene 4222

Handlungs- vs. Lageorientierung 133

Hypertonie 143

Ich-Dystonie 3162

Ich-Syntonie 3162

«ldeot Savant» 4234

Image der Verhaltenstherapie 294

Imagination 3124 Imaginative Verfahren 3124 Individualisierung von Therapie 4244 Inkontinenz 143

Jugendliche 288

Katamneseuntersuchung 122 Klinische Psychologie und

Verhaltenstherapie 3170 Kognitive Verhaltenstherapie 133 Konsiliar-/Liaisontätigkeit 122 Kurzzeitintervention 122

Mentale Retardierung 4234 Metaanalyse 14 Migräne 4210

Neurophysiologie 4210

Öffentliches Gesundheitswesen 3170

Ost-West-Unterschiede 288

Panik 114 Paniksyndrom 3135 Paradigmawechsel 4244 Partnerkonflikt 114 Pathogenese 4210 Persönlichkeitsstörungen 4201 Pilotprojekt 4201

Plananalyse 133 Prävalenz 4222

Psychosoziale Interventionsstudien 14 Psychotherapeutenausbildung, praxisnahe 2107

Qualitätssicherung 3170

Schizophrenie 14, 4201 Schizotypie 288 Selbsthilfegruppen 3143 Selbstwirksamkeit 143 Somatoforme Störungen 122,143 Standardtherapie 4244 Stationäre Behandlung 4201 Stimulantien 4222 Störungsmodell-Heuristik 4244 Styles of Conflict Inventory 114 Sucht 4201 Supervision 2107

Therapieforschung 3135

Uberprüfung von Therapeutenverhalten

3135

Verhaltensmedizin 122,2 76,4 210 Verhaltenstherapeut (Selbstbild;

Fremdbild - Psychoanalytiker) 294 Videofeedback 3162 Vorstellungsfähigkeit 3124 Vorstellungstraining 3124

Wissenschaftler-Praktiker-Modell 2107

Zwanghafte Persönlichkeitsstörung

3162 Zwangsstörung 3 143, 3162

275 